\title{
Image Inpainting Based on Sparse Representation with Histogram Dictionary
}

\author{
Qiaoqiao Li1 ${ }^{*}$, Guoyue Chen ${ }^{2}$, Xingguo Zhang², Kazuki Saruta², Yuki Terata ${ }^{2}$ \\ ${ }^{1}$ The Graduate School of Systems Science and Technology, Akita Prefectural University, Akita, Japan. \\ ${ }^{2}$ Dept. of Electronics and Information Systems, Akita Prefectural University, Akita, Japan. \\ * Corresponding author. Email: liqiaoqiao098@gmail.com \\ Manuscript submitted May 10, 2018; accepted July 8, 2018. \\ doi: $10.17706 / j c p .13 .10 .1145-1155$
}

\begin{abstract}
This paper proposes a novel image inpainting algorithm based on sparse representation with histogram dictionary. In the propose method, it is a patch-based inpainting method, a patch as the basic unit of inpainting algorithm. Instead of using all the known image patches to form a dictionary, we propose to construct a histogram dictionary by using the histogram. If the histogram between an image patch which cuts from the source region of the input image and the target patch are similar, the image patch as the similar patch is selected to generate a similar dictionary for inpainting algorithm. Two kinds of similarity methods are proposed, so two kinds of histogram dictionaries are corresponding. These histogram dictionaries can ensure a large related to the target patch. Experimental results show that the proposed inpainting method using the histogram dictionary can efficiently inpainting of missing areas.
\end{abstract}

Key words: Image inpainting, sparse representation, histogram dictionary, similarity.

\section{Introduction}

The filling-in of missing or corrupted region in an image, which is cased image inpainting and is an inverse ill-posed problem, is an import topic in the field of computer vision and image processing [1]-[7]. Image inpainting, has been extensively investigated in the applications of digital effect (e.g., object removal), image coding and transmission (e.g., recovery of the missing block), image restoration (e.g., scratch or text removal in photograph), etc. [1]-[3].

Many approaches for image inpainting problem have been proposed [1]-[3], [5]-[14]. Generally, these methods are categorized into three categories: diffusion-based inpainting approach, exemplar-based inpainting approach, and inpainting approach based on sparse representation[1], [3], [6]. The first category is named as diffusion-based inpainting methods, which are based on the parametric model or partial differential equation (PDE). Bertalmio et. al [1] started to use the anisotropic diffusion partial differential equations (PDF) for image inpaitning. Once the user selects the region to be restored, they can use the information surrounding the missing region to restore them by using information propagation. The directions of the propagation are based on the direction of the isophotes. Considering the image intensity corresponds to a stream in fluid dynamics and isophote is correspond to flow streamlines, they also proposed a method of image inpainting by using the Navier-Strokes equation in fluid dynamics [6]. Chan and Shen [7] proposed a model based on Total variation (TV) for image inpainting. Although these diffusion-based inpainting methods perform well for filling the non-texture or relatively smaller missing 
region, some blur will be introduced in the texture or large missing region.

In the exemplar-based inpainting method, this kind of methods are inspired by the idea of texture synthesis technique [8]. These algorithms see the image patch as a unit, and the basic idea of these algorithms is finding the best match patch to inpaint the missing areas. As we all known, a natural image is composed of structural information suitable and texture information. According this fact, Bertalmio et.al [9] proposed a method which decomposes the image into structure layer and texture layer. Then they employed the method of diffusion [1] to inpaint the structure layer, and used the technique [8] to process the texture layer. After that, the two reconstructed images are added back together to obtain the inapitning result. Criminisi et. al [10], [11] proposed an exemplar-based inpainting algorithm, in order to preserve the structure information they defined image patch priority. Le Meur et. al [12] improved the filling order based on structure tensor, and applied the new filling order for their exemplar-based inpainting. Xu et.al [13] proposed a method by patch propagation by using a modified filling-in order and inpainted the missing area using the sparse linear combination of candidate patches. Deng et. al [14] proposed an exemplar-based image inpainting method by using a new priority definition which separates the Criminisi's priority definition into two phases. However, the traditional exemplar-based methods which find a best match patch and copy pixel values to restore the corrupted patch directly. It can cause the unwanted information to the inpainting patch. Moreover, searching the whole image to find a best match patch is time consuming.

Recently, sparse representation has introduced into image inpainting [2], [5], [15]-[20]. The main point of this method is to represent the image patches by using a sparse linear combination of atoms from a dictionary. Thus, the dictionary is very important for inpainting results. Amano et. al [18] utilized the learning samples which were clipped from the local region (where has no defect region) as eigenvector series in their interpolation method and obtained the good image interpolation results. Shen et. al [19] directly used all the patches in the source region to construct a dictionary and obtained a good inpainting results. However, the dictionary contains all the patches which are in the source region, it will have some of unrelated atoms with an image patch to be restored. Moreover, the unrelated atoms will introduce the interference into inpainting results. Aharon et. al [20] proposed a K-SVD algorithm for designing dictionaries for sparse presentation. Mairal et. al [16] introduced a sparse representation for color image restoration by using the K-SVD algorithm. However, this method is suitable for denoising, but is not suitable for inpainting.

In this paper, we aim is to improve the design of a dictionary, and propose an image inpainting method based on sparse representation. The proposed method also uses image patch as the basic unit of sparse representation. Specifically, we don't simply cut the patches from the known region to form a dictionary, but compare the similarity between the target patch which we want to inpaint, and the whole candidate patches in the known region by using the histogram. And then, we can use similar patches, some parts of the whole candidate patches, to generate a histogram dictionary. We here introduce two kinds of similarity methods, so two kinds dictionaries are corresponding. Instead of only using the best match candidate patch [10], [11], we utilize some patches which are related to the target patch for image inpainting. This can avoid the interference of irrelevant patches effectively. The proposed image inpainting method is compared with state-of-the-art methods, experimental results shown the efficiency of the proposed inpainting method. This paper is structured as follows. Section 2, introduces a brief description of sparse representation. Section 3, describes the histogram dictionary with two similar methods. Section 4, presents the sparse representation-based inpainting algorithm. Section 5, shows our experimental results. Section 6, concludes this paper with a brief description. 


\section{Preliminaries}

Recently, signal sparse representation has attracted many researches' attention. And a patch is always set to a basic unit in signal sparse representation [5], [20], [21].

Given a dictionary $\boldsymbol{D}$ whose columns are prototype signal-atoms atoms $\boldsymbol{D}=\left[d_{1}, \cdots, d_{k}\right]$, a patch $\boldsymbol{y}$ can be represented as a sparse linear combination of these atoms, that is,

$$
\boldsymbol{y}=\boldsymbol{D} \boldsymbol{\alpha}
$$

where $\alpha$ are the sparse representation coefficients of the patch.

Considering the inpainting problem, some pixels of the image patch are damaged. Therefore, we can divide the patch $\boldsymbol{y}$ into the known pixels and missing pixels, respectively. Denote $\boldsymbol{M}$ as a matrix to extract the known pixels in $y$

$$
\mathbf{y}^{*}=\mathbf{M y}
$$

In the problem of image inpainting, we want to use the known pixels $\mathbf{y}^{*}$ to estimate the patch $\boldsymbol{y}$. First, infer the sparse coefficients $\alpha$ by minimizing an optimization problem

$$
\min _{\boldsymbol{\alpha}}\|\boldsymbol{\alpha}\|_{0} \quad \text { s.t. } \quad \mathbf{y}^{*}=\mathbf{M D} \boldsymbol{\alpha}
$$

where \|\|$_{0}$ means $l_{0}$ norm which counts the nonzero elements in $\boldsymbol{\alpha}$. Because the problem of $l_{0}$ norm is usually an NP-hard problem [22], thus it is often solved by greedy algorithms such as matching pursuit (MP) [23] and the orthogonal matching pursuit (OMP) [24] algorithms.

Second, after obtaining the estimation of coefficients $\hat{\alpha}$, the image patch $y$ can be reconstructed from its sparse coefficients $\hat{\alpha}$ by using

$$
\hat{\boldsymbol{y}}_{i}=\left\{\begin{array}{lrr}
\mathbf{y}_{\mathbf{i}}^{*}, & \text { if } & i \in \mathbf{y}^{*} \\
\overline{\mathbf{M}} \mathbf{D} \hat{\boldsymbol{\alpha}}, \text { if } & i \notin \mathbf{y}^{*}
\end{array}\right.
$$

where $\bar{M}$ is a matrix which is determined the location of missing pixels.

\section{Histogram Dictionary}

In this section, we will introduce how to flexibly construct a histogram dictionary for a target patch $\Psi_{\mathrm{p}}$ in the given image $\boldsymbol{I}$.

First, select a target patch $\boldsymbol{\Psi}_{\mathrm{p}}$ using the filling order which is described by Criminisi et. al [10], [11] and compute the histogram of it. Let the histogram have $\mathrm{N}$ bins. Therefore, the histogram of color image has $\mathrm{N}$ values. For three channels R, G, B of target patch $\Psi_{p}$, the histogram of each channel can be represented by

$$
\begin{aligned}
\mathbf{h}_{\boldsymbol{\Psi}_{\mathrm{p}} \mathrm{R}} & =\left[h_{\boldsymbol{\Psi}_{\mathrm{p}} \mathrm{R} 1}, \cdots, h_{\boldsymbol{\Psi}_{\mathrm{p}} \mathrm{R} N}\right]^{T} \\
\mathbf{h}_{\boldsymbol{\Psi}_{\mathrm{p}} \mathrm{G}} & =\left[h_{\boldsymbol{\Psi}_{\mathrm{p}} \mathrm{G} 1}, \cdots, h_{\boldsymbol{\Psi}_{\mathrm{p}} \mathrm{G} N}\right]^{T} \\
\mathbf{h}_{\boldsymbol{\Psi}_{\mathrm{p}} \mathrm{B}} & =\left[h_{\boldsymbol{\Psi}_{\mathrm{p}} \mathrm{B} 1}, \cdots, h_{\boldsymbol{\Psi}_{\mathrm{p}} \mathrm{B} N}\right]^{T}
\end{aligned}
$$

Second, cut the whole known patches $f_{i}(i=1, \ldots, 1)$ from the image $I$ and compute the histogram of them (with $L$ denotes the number of known patches). $f_{\mathrm{R} i}, f_{\mathrm{G} i}$ and $f_{\mathrm{B} i}$ denote three channels (RGB) of the patch, respectively. The histogram of each channel can be represented by

$$
\begin{gathered}
\mathbf{h}_{\mathrm{f}_{\mathrm{Ri}}}=\left[h_{f_{\mathrm{Ri1}}}, \cdots, h_{f_{\mathrm{R}, \mathrm{N}}}\right]^{T} \\
\mathbf{h}_{\mathrm{f}_{\mathrm{Gi}}}=\left[h_{f_{\mathrm{Gi}}}, \cdots, h_{f_{\mathrm{GiN}} \mathrm{N}}\right]^{T} \\
\mathbf{h}_{\mathrm{f}_{\mathrm{Ei}}}=\left[h_{f_{\mathrm{Bi}}}, \cdots, h_{f_{\mathrm{BiN}}}\right]^{T}
\end{gathered}
$$

Thus, two patches $\Psi_{\mathrm{p}}$ and $f_{i}$ can use the simplest method to measure similarity by comparing 
corresponding bins of the histogram. In $R, G, B$ three channels, the difference can be defined by comparing their histogram as follows:

$$
\begin{gathered}
\boldsymbol{v}_{\boldsymbol{R} i}=\left\|\mathbf{h}_{\boldsymbol{\Psi}_{\mathrm{pR}}}-\mathbf{h}_{\mathrm{f}_{\mathrm{R} i}}\right\|_{1}=\sum_{j=1}\left(\left|h_{\Psi_{p R j}}-h_{f_{\mathrm{R} i j}}\right|\right) \\
\boldsymbol{v}_{\boldsymbol{G} i}=\left\|\mathbf{h}_{\boldsymbol{\Psi}_{\mathrm{p}} \mathbf{G}}-\mathbf{h}_{\mathbf{f}_{\mathrm{Gi}}}\right\|_{1}=\sum_{j=1}^{N}\left(\left|h_{\Psi_{p} \mathrm{Gj}}-h_{f_{\mathrm{G} i j}}\right|\right) \\
\boldsymbol{v}_{\boldsymbol{B} i}=\left\|\mathbf{h}_{\boldsymbol{\Psi}_{\mathrm{p}} \mathrm{B}}-\mathbf{h}_{\mathrm{f}_{\mathrm{Bi}}}\right\|_{1}=\sum_{j=1}^{N}\left(\left|h_{\Psi_{p} \mathrm{~B} j}-h_{f_{\mathrm{B} i j}}\right|\right)
\end{gathered}
$$

The aim is to find out the similar patches from the whole candidate patches, so how to select the similar patch is a problem.

In order to solve this problem, two similarity method by using histogram are proposed.

\subsection{Similarity Based on Sum}

The similarity based on sum $v_{S i}$ is defined as

$$
v_{S i}=v_{R i}+v_{G i}+v_{B i}
$$

Considering the number of the known patches is 1 , the similarity based on sum is written by

$$
v_{S}=\left[v_{\mathrm{s} 1}, \cdots, v_{S l}\right]^{T}
$$

Then, sort the $v_{S}$ and find the top $t(t<1)$ known patches to generate the dictionary.

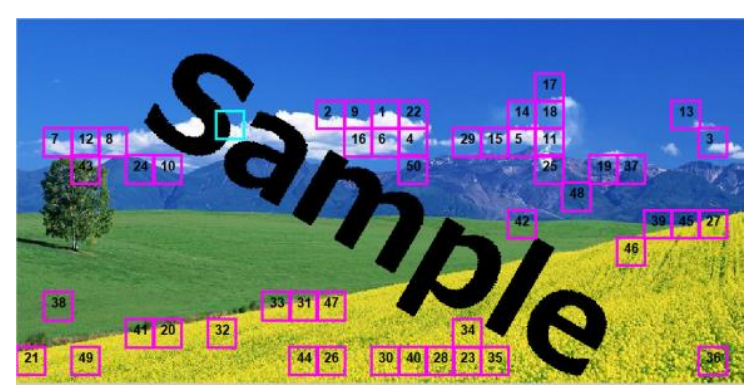

a. Target patch 1 and its chosen patches

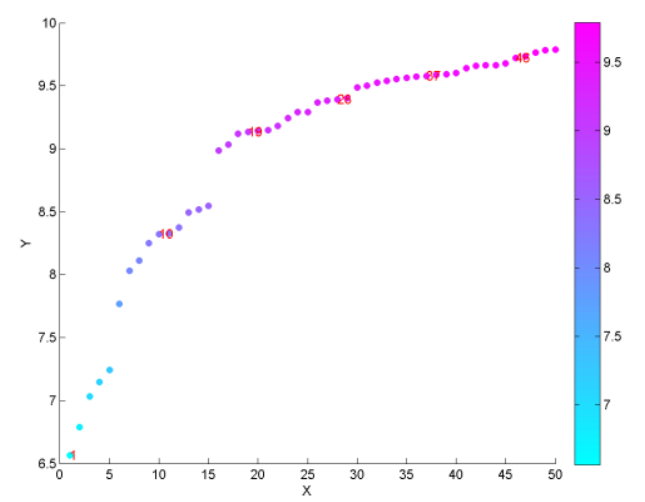

c. Chosen patches of (a) are shown in scatter plots

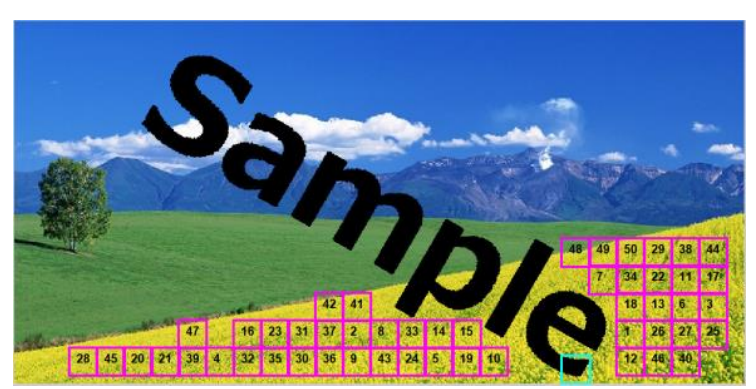

b. Target patch 2 and its chosen patches

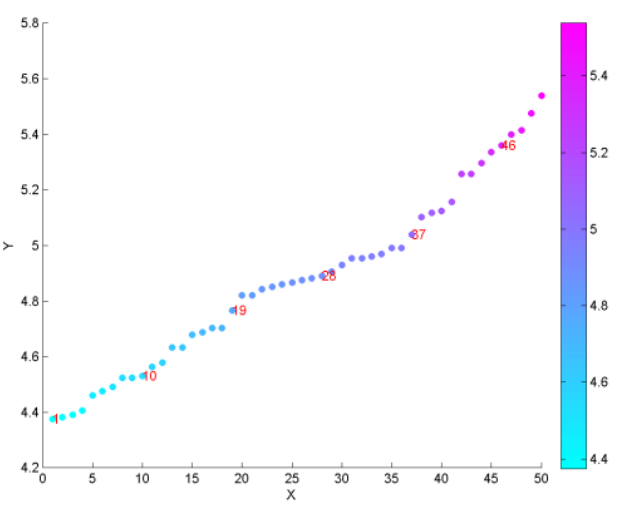

d. Chosen patches of (b) are shown in scatter plots

Fig. 1. The chosen patches by using sum histogram.

In order to see clearly which patches are chosen for generating the dictionary, we mark out the top $t=50$ most similar sample patches which are used to construct a histogram dictionary. That is to say, the sample patch 1 is the first similar to the target patch and so on. The result is shown in Fig. 1. Fig. 1 (a) and Fig. 1 (b) are shown one target patch (blue-green) and its top 50 chosen patches (mulberry). Fig. 1 (c) and Fig. 1 (d) are shown the chosen patches of the two samples by using the similarity based on sum in scatter 
plots.

\subsection{Similarity Based on Max}

Considering the three channels of the color image, we see the similarity of patches as a 3-D vector and find the maximum value of it. The similarity based on $\max v_{M i}$ is defined as

$$
v_{M i}=\max \left(v_{R i}, v_{G i}, v_{B i}\right)
$$

Considering the number of the known patches is $L$, the similarity based on max is written by

$$
v_{M}=\left[\begin{array}{lll}
v_{\mathrm{M1}}, & \cdots & , v_{M l}
\end{array}\right]^{T}
$$

Then, sort $v_{M}$ and find the top $t(t<l)$ known patches to generate the dictionary.

For 3-D case, in Fig. 2 (a) -(b) are shown the top $t=50$ sample patches which are constructed a histogram dictionary for the two target patches by comparing similarity based on max. In Fig. 2 (c) -(d) are shown the chosen patches of the two samples by using similarity based on max in scatter plots.

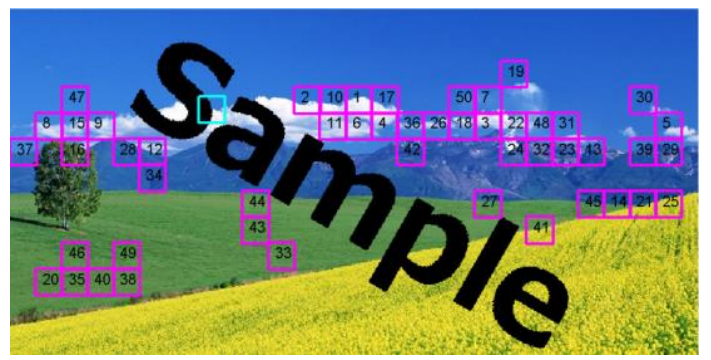

a. Target patch 1 and its chosen patches

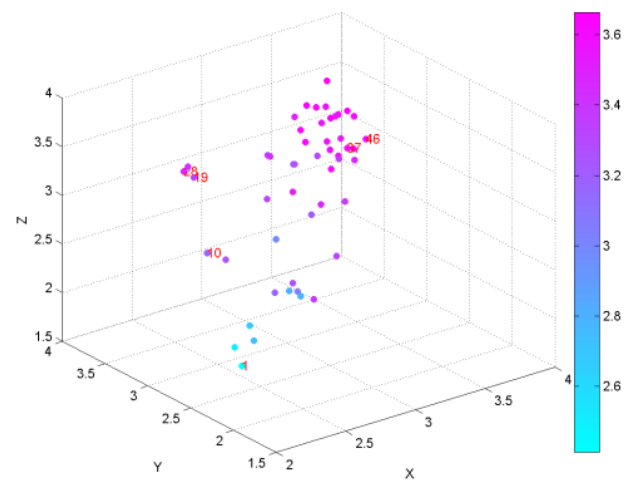

c. Chosen patches of (a) are shown in scatter plots plots

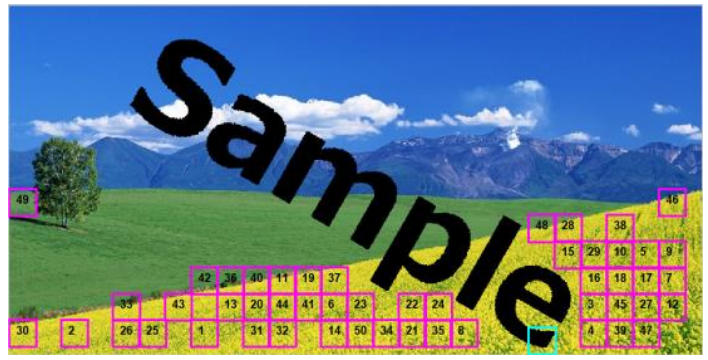

b. Target patch 1 and its chosen patches

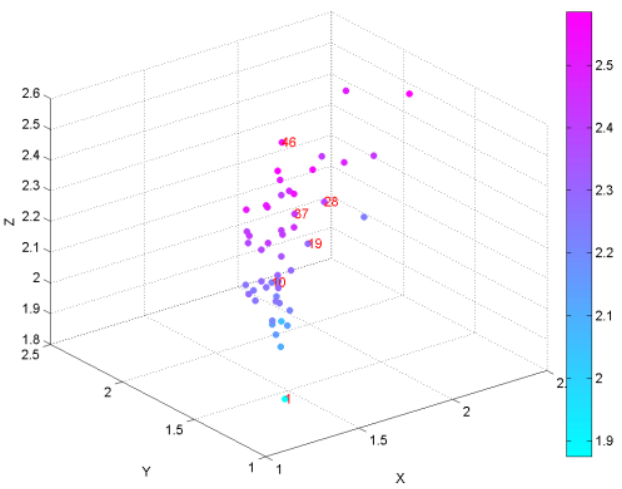

d. Chosen patches of (b) are shown in scatter

Fig. 2. The chosen patches by using max histogram.

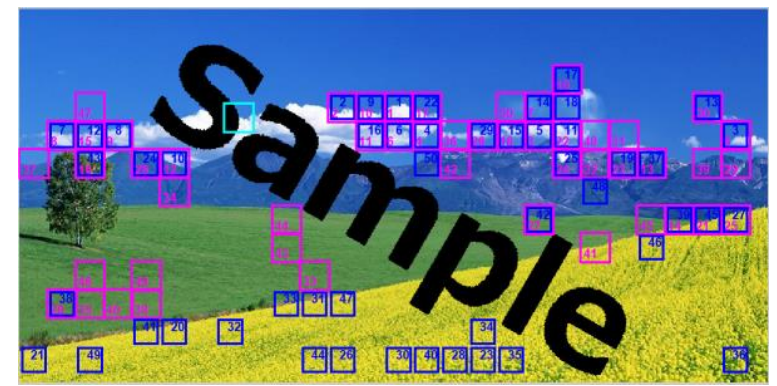

a. Target patch 1 and its chosen patches by using two similarity methods

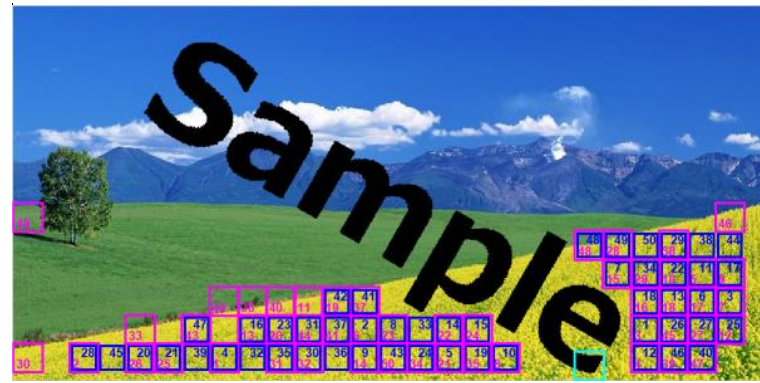

b. Target patch 2 and its chosen patches by using two similarity methods

Fig. 3. The chosen patches with two similar methods are shown in the same picture. 
Using the two different methods to comparing the similarity, the histogram dictionary will be different. In order to view the patches are chosen to form histogram dictionary with comparing similarity method easily, Fig. 3 is shown the chosen top 50 patches with the two similarity methods in the same picture. The mulberry block is shown the results of based on max method and the blue block are shown the based on sum method, respectively. From Fig. 3 (a), the chosen top 50 sample patches by using similarity based on max contain more relevant patches than that by using similarity based on sum. From Fig. 3 (b), the chosen top 50 sample patches by using similarity based on max are generally as relevant as the chosen patches by using similarity based on sum.

\section{Overview of the Proposed Algorithm}

Furthermore, a new image inpainting method is proposed based on this histogram dictionary. As discussed above, the schematic diagram of the proposed inpainting algorithm is shown in Fig. 4.

The detailed procedures of the proposed method are as follows:

First, input the inpainting image which is denoted by $\boldsymbol{I}$. And the image $\boldsymbol{I}$ contains the source region $\boldsymbol{S}$ (undamaged region) and the target region $\boldsymbol{T}$ (damaged region). That is to say

$$
\boldsymbol{I}=\boldsymbol{S}+\boldsymbol{T}
$$

Second, provide a window size of $\mathrm{n} \times \mathrm{n}$ and cut the patches from the source region $S$, and then a group of known patches $\left\{\boldsymbol{f}_{i}\right\}_{i=1}^{l}$ can be obtained.

Third, use the filling order method proposed by Criminisi et. al [10], [11] to choose the target patch $\Psi_{\mathrm{p}}$, as the beginning patch for our proposed method. The window size is same as the second step. The known pixels can be obtained by

$$
\Psi_{\mathrm{p}}^{*}=\boldsymbol{M} \boldsymbol{\Psi}_{\mathrm{p}}
$$

Fourth, compare the similarity between the target patch and the all known patches by using the methods which are described in the section III. And then select the $m$ similar patches to create a histogram dictionary for the target patch. The size of histogram dictionary is $n^{2} \times m \quad(\mathrm{~m}<1)$ which is denoted by $\boldsymbol{D}_{\boldsymbol{h}}$. Because there are two similar methods for proposed inpainting method, the histogram dictionaries $\boldsymbol{D}_{\boldsymbol{h}}$ are also two kinds. The histogram dictionary using similarity based on sum is denoted by $\boldsymbol{D}_{h s}$ and histogram dictionary using similarity based on sum is denoted by $\boldsymbol{D}_{h M}$, respectively.

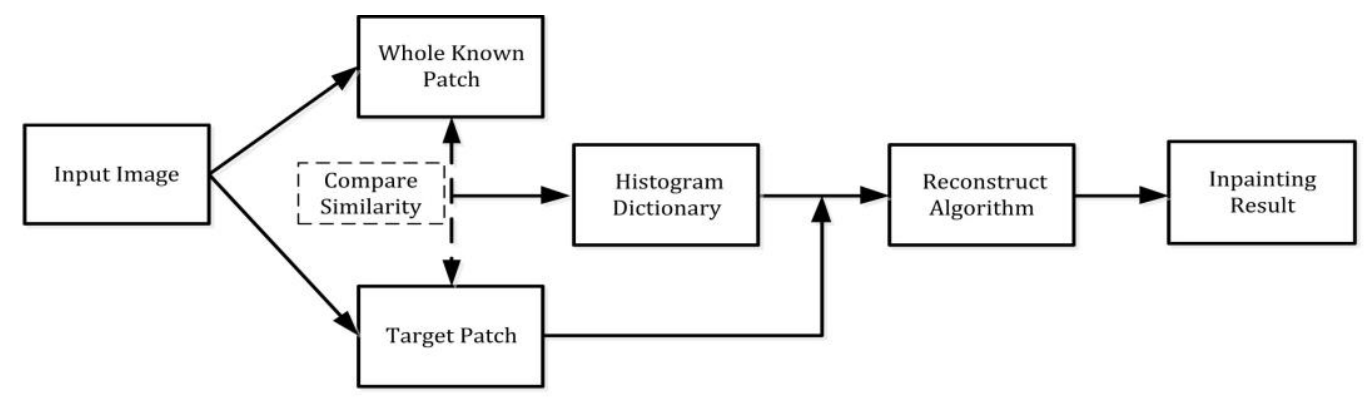

Fig. 4. Schematic diagram for the proposed inpainting algorithm.

Finally, estimate the sparse coefficients $\hat{\alpha}$ by using the non-negative orthogonal matching pursuit algorithm (NNOMP) [25] which is an improved method of OMP [24]. Reconstructing the target patch $\Psi_{\mathrm{p}}$ from the sparse coefficients $\hat{\alpha}$ is expressed as: 


$$
\hat{\mathbf{\Psi}}_{\mathbf{p}}^{\mathbf{i}}=\left\{\begin{array}{lll}
\boldsymbol{\Psi}_{\mathrm{p}}^{\mathbf{i}}, & \text { if } & i \in S \\
\overline{\mathbf{M}} \mathbf{D}_{\mathbf{h}} \hat{\boldsymbol{\alpha}}, & \text { if } & i \in \boldsymbol{T}
\end{array}\right.
$$

\subsection{Image Inapainting Algorithm}

In the light of introduction above, the proposed method based on sparse representation by using histogram dictionary is described in detail in Algorithm 1. Here, we adopt the histogram dictionary $\boldsymbol{D}_{h M}$ by using the similarity based on max to explain. For the histogram dictionary $\boldsymbol{D}_{h \mathrm{~S}}$ just replacing the similarity based on sum $v_{M}$ to the similarity based on $\operatorname{sum} v_{S}$, the process is same.

Algorithm 1 The proposed method based on sparse representation by using histogram dictionary

1: Input: the observed image $I$

2: initialization: block $=n \times n, \boldsymbol{K P}, l=0$

3: Repeat:

4: clipped the patch $\mathbf{f}_{l+1}$ from the image $\boldsymbol{I}$ in a window $(n \times n)$

5: if $\mathbf{f}_{l+1}$ is in the source region $S$ of the image $I$

6: $\mathbf{f}_{l+1}$ is as a candidate patch

7: compute the histogram of the patch by equation (6)

8: $l=l+1$

9: update $\mathbf{K P}_{\mathbf{l + 1}}=\left[\begin{array}{ll}\boldsymbol{K} \boldsymbol{P}_{l} & \mathbf{f}_{\mathbf{l + 1}}\end{array}\right]$

10: end if

11: until

12: Iteration: the window scans the bottom right corner

13: while $I$ has defective pixels do

14: initialization: $v_{M}=[$ ]

15: $\quad$ use the filling order to find the target patch $\Psi_{\mathrm{p}}$

16: compute the histogram of it by equation (5)

17: for $i=1: l$

18: compute the difference between the target patch and the candidate patches by equation (7)

19: use $\quad v_{M i}=\max \left(v_{R i}, v_{G i}, v_{B i}\right)$

20: update $v_{M}=\left[\begin{array}{ll}v_{M} & v_{M i}\end{array}\right]$

21: end for

22: $\quad$ sort the maximum value by

23: $\quad$ index $=\operatorname{sort}\left(\boldsymbol{v}_{M}\right)$

24: $\quad$ obtain the histogram dictionary by

25: $\quad \boldsymbol{D}_{\boldsymbol{h}}=\boldsymbol{K P}(:, \operatorname{index}(1: l))$

26: $\quad$ estimate the sparse coefficient by

27: $\hat{\boldsymbol{\alpha}}=\operatorname{FNNOMP}\left(\boldsymbol{\psi}_{\mathrm{p}}, \boldsymbol{D}_{\boldsymbol{h}}\right)$

28: reconstruct the $\psi_{\mathrm{p}}$ by using equation (14)

\section{9: end while}

30: Output: inpainting image $\hat{\boldsymbol{I}}$

\section{Experiment Results}

In this part, we test the performance of the proposed method on a variety of natural images. We compare 
the proposed method with Criminisi's [10], [11] inpainting algorithm. The proposed method is also employs the inpainting filling order which is described in [10], [11]. And the proposed method is implemented by using Algorithm 1 based on the schematic diagram in Fig. 5. The number of $L$ is set as 400, that is to say we choose the top 400 patches as the similar dictionary. For fairness, the window size of all methods are set $9 \times 9$.

The performance of the proposed method is compared with different image inpainting methods in quantitative evaluations. We use the peak signal-to-noise ratio (PSNR) as the metrics to evaluate the imapinting results. Furthermore, in order to see clearly, PSNR values in three channels (R, G, B) are also presented. The larger the value of the PSNR, the better the inpainting result.
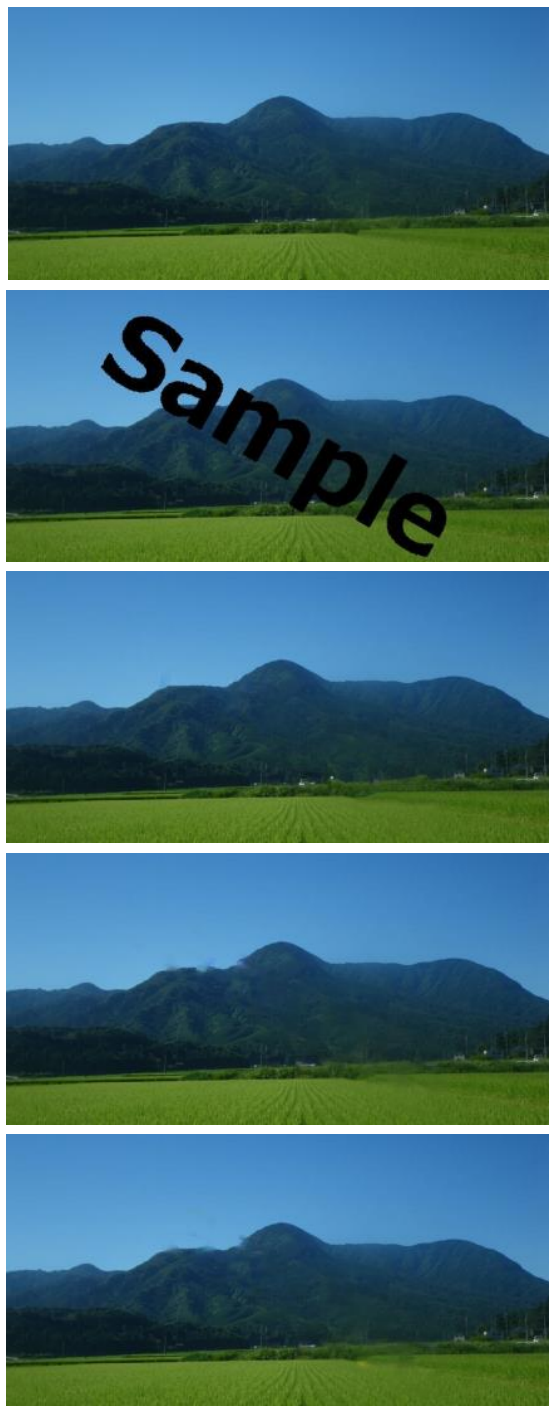

a
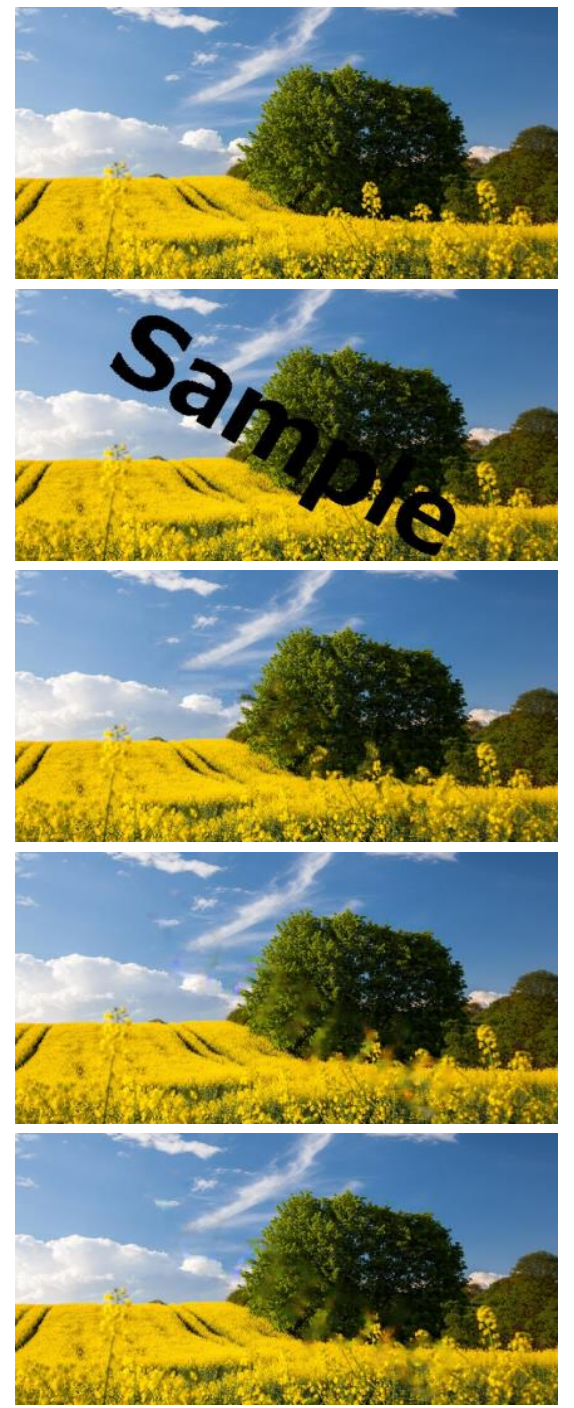

b
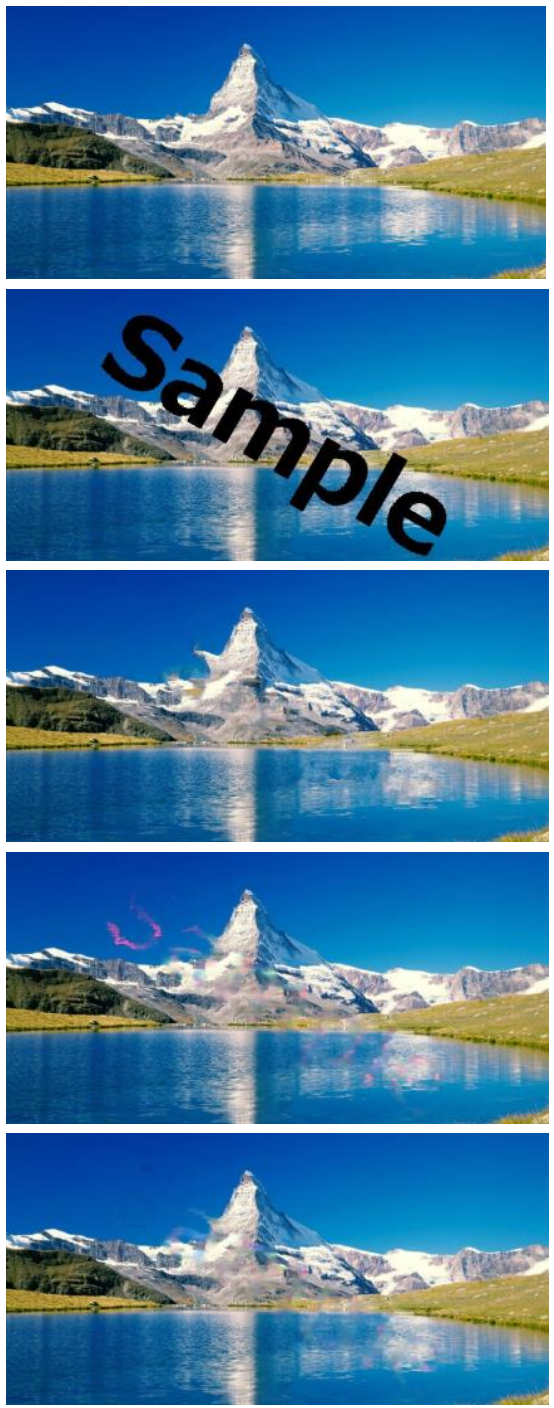

C

Fig. 5. Obtained results of three natural images. The first row shows three original images. The second row shows the degraded images. The third to fifth row show the result of Criminisi, the proposed method based on histogram dictionary $\boldsymbol{D}_{h S}$ and the proposed method based on histogram dictionary $\boldsymbol{D}_{\boldsymbol{h} M}$.

Considering the two similar methods, the proposed method inpaint the missing region by using the histogram dictionary $\boldsymbol{D}_{h S}$ and $\boldsymbol{D}_{h M}$. Fig. 6 presents three testing images for this experiment. The first row is the original noncorrupted images. In the remaining rows, from the first and the fourth rows are the 
corrupted images, results of Criminisi's inpainting algorithm [10], [11], the proposed method by using the histogram $\boldsymbol{D}_{h S}$ and the proposed method by using the histogram $\boldsymbol{D}_{h M}$. The peak signal-to-noise ratio (PSNR) between the result and the original image is summarized in Table 1. It can be seen in Fig. 6, the Criminisi's algorithm cause obvious miscopies in the third row. For instance, the snow mountain in (c) of the third row appears unwanted structure of the Criminis's result. This is because the Criminisi's method only chooses a best match patch to inpaint missing region, some unwanted artifacts appear the results. In the fourth row, the result of the proposed method by using the histogram dictionary $\boldsymbol{D}_{h S}$ is shown. The edge of the mountain is not inpainted very well in (a) of the fourth row. And in (c) of the fourth row, extra color produces in the result. For the proposed method by using the histogram dictionary $\boldsymbol{D}_{h M}$, the similar patches are chosen by similarity based on max in the framework of sparse representation, so it can overcome the influences which caused by Criminisi's inpainting method. Furthermore, the histogram dictionary $\boldsymbol{D}_{\boldsymbol{h} M}$ is generated by comparing the difference in 3-D vector, it is more suitable for the color image than the histogram dictionary $\boldsymbol{D}_{h S}$ for the proposed method. And the fact confirms the quantitative metrics as shown in Table 1. The objective quantitative evaluation is consistent with the subjective visual effect of the inpainting result images.

Table 1. Objective Performance

\begin{tabular}{llccc}
\hline \hline Image & RGB & Criminisi & Sum & Max \\
\hline \multirow{2}{*}{ Image 1 } & $\mathrm{R}$ & 38.8751 & 40.8970 & 41.3303 \\
& $\mathrm{G}$ & 38.4905 & 39.7892 & 40.7463 \\
& $\mathrm{~B}$ & 39.0629 & 39.9818 & 40.9369 \\
& $\mathrm{RGB}$ & 38.8095 & 40.2227 & $\mathbf{4 1 . 0 0 4 5}$ \\
\hline \multirow{2}{*}{ Image 2 } & $\mathrm{R}$ & 24.3601 & 25.5961 & 25.6186 \\
& $\mathrm{G}$ & 26.0846 & 27.3549 & 27.3753 \\
& $\mathrm{~B}$ & 32.5088 & 32.1619 & 33.7642 \\
& $\mathrm{RGB}$ & 27.6512 & 28.3710 & $\mathbf{2 8 . 9 1 9 4}$ \\
\hline \multirow{3}{*}{ Image 3 } & $\mathrm{R}$ & 24.4232 & 24.4091 & 26.7018 \\
& $\mathrm{G}$ & 24.8705 & 30.2230 & 30.2815 \\
& $\mathrm{~B}$ & 29.2284 & 32.2277 & 32.1201 \\
\hline \hline
\end{tabular}

\section{Conclusion}

In this paper, we have proposed a new inpainting method based on sparse representation. In this paper, two measure similarity methods, i.e. based on sum and similarity based on max, are proposed for comparing the similarity between the target patch and the all candidate patches. And then the similar patches are chosen to form a histogram dictionary. In this way, the interference of the non-related patches to the sparse construction to be avoided. The proposed inpainting method using the histogram dictionary shows good performance both in the PSNR sense and visually when compared with the Criminisi's method.

\section{Acknowledgment}

This work was supported by the China Scholarship Council (CSC 201708050084).

\section{References}

[1] Bertalmio, M., Sapiro, G., Caselles, V., \& Ballester, C. (2000). Image inpainting. Proceedings of the 27th Annual Conference on Computer Graphics and Interactive Techniques (pp. 417-424). 
[2] Fadili, M. J., Starck, J. L., \& Murtagh, F. (2007). Inpainting and zooming using sparse representations. The Computer Journal, 52(1), 64-79.

[3] Guillemot, C., \& Meur, O. L. (2014). Image inpainting: Overview and recent advances. IEEE Signal Processing Magazine, 31(1), 127-144.

[4] Zhang, J., Zhao, D., \& Gao, W. (2014). Group-based sparse representation for image restoration. IEEE Transactions on Image Processing, 23(8), 3336-3351.

[5] Xu, K., Wang, N., \& Gao, X. (2016). Image inpainting based on sparse representation with dictionary pre-clustering. Proceedings of Chinese Conference on Pattern Recognition. Springer (pp. 245-258).

[6] Bertalmio, M., Bertozzi, A. L., \& Sapiro, G. (2001) Navier-stokes, fluid dynamics, and image and video inpainting. Proceedings of the 2001 IEEE Computer Society Conference on Computer Vision and Pattern Recognition.

[7] Chan, T., \& Shen, J. (2001). Local inpainting models and tv inpainting. SIAM J. Appl. Math, 62(3), 1019-1043.

[8] Efros, A. A., \& Leung, T. K. (1999). Texture synthesis by non-parametric sampling in computer vision. Proceedings of the Seventh IEEE International Conference (pp. 1033-1038).

[9] Bertalmio, M., Vese, Sapiro, L. G., \& Osher, S. (2003). Simultaneous structure and texture image inpainting. IEEE Transactions on Image Processing, 12(8), 882-889.

[10] Criminisi, A., Perez, P., \& Toyama, K. (2004). Region filling and object removal by exemplar-based image inpainting. IEEE Transactions on Image Processing, 13(9), 1200-1212.

[11] Criminisi, A., Perez, P., \& Toyama, K. (2003). Object removal by exemplar-based inpainting. Proceedings of IEEE Computer Society Conference on Computer Vision and Pattern Recognition (pp. II-II) .

[12] Meur, O. Le., Gautier, J., \& Guillemot, C. (2011). Examplar-based inpainting based on local geometry. Proceedings of the 18th IEEE International Conference on Image Processing (ICIP) (pp. 3401-3404).

[13] Xu, Z., \& Sun, J. (2010). Image inpainting by patch propagation using patch sparsity. IEEE Transactions on Image Processing, A Publication of the IEEE Signal Processing Society, 19(5), 1153-1165.

[14] Deng, L. J., Huang, T. Z., \& Zhao, X. L. (2005). Exemplar-based image inpainting using a modified priority definition. Plos One, 10(10).

[15] Elad, M., Starck, J. L., Querre, P., \& Donoho, D. L. (2005). Simultaneous cartoon and texture image inpainting using morphological component analysis (mca). Applied and Computational Harmonic Analysis, 19(3), 340-358.

[16] Mairal, J., Elad, M., \& Sapiro, G. (2008). Sparse representation for color image restoration. IEEE Transactions on Image Processing, 17(1), 53-69.

[17] Ogawa, T., \& Haseyama, M. (2013). Image inpainting based on sparse representations with a perceptual metric. EURASIP Journal on Advances in Signal Processing, 2013(1), 179.

[18] Amano, T., \& Sato, Y. (2007). Image interpolation using bplp method on the eigenspace. Systems and Computers in Japan, 38(1), 87-96.

[19] Shen, B., Hu, W., Zhang, Y., \& Zhang, Y.-J. (2009). Image inpainting via sparse representation. Proceedings of IEEE International Conference on Acoustics, Speech and Signal Processing (pp. 697-700).

[20] Aharon, M., Elad, M., \& Bruckstein, A. (2006). rmk-svd: An algorithm for designing overcomplete dictionaries for sparse representation. IEEE Transactions on Signal Processing, 54(11), 4311-4322.

[21] Elad, M., \& Aharon, M. (2006). Image denoising via sparse and redundant representations over learned dictionaries. IEEE Transactions on Image processing, 15(12), 3736-3745.

[22] Davis, G., Mallat, S., \& Avellaneda, M. (1997). Adaptive greedy approximations. Constructive 
Approximation, 13(1), 57-98.

[23] Mallat, S., \& Zhang, Z. (1993).Matching pursuits with time-frequency dictionaries. IEEE Transactions on Signal Processing, 41(12), 3397-3415.

[24] Tropp, J. A., \& Gilbert, A. C. (2007). Signal recovery from random measurements via orthogonal matching pursuit. IEEE Transactions on Information Theory, 53(12), 4655-4666.

[25] Yaghoobi, M., Wu, D., \& Davies, M. E. (2015). Fast non-negative orthogonal matching pursuit. IEEE Signal Processing Letters, 22(9), 1229-1233.

Qiaoqiao Li received the B. Eng. degree from Zhengzhou University, Zhengzhou, China, in 2012, and the M. Eng. degree from Lanzhou University, Lanzhou, China, 2016. She is currently pursuing Dr. Eng. degree at Akita Prefectural University, Akita, Japan. Her research interests include signal processing and image Processing.

Guoyue Chen received his B.Sc degree from East China Normal University, China, in 1983, where he worked as a research associate at the Department of Computer Science until 1989. He received his M.S and Ph.D degrees from Tohoku University, Japan in 1993 and 1996, respectively. He is currently a professor in Akita Prefecture University, Japan. His interests include digital signal processing and its applications to active noise control, and image processing.

Xingguo Zhang received the B.E. degree in computer science from the Dalian Polytechnic University of China in 2009, and the M.E and Ph.D degrees from the Akita Prefectural University of Japan in 2012 and 2015. Currently, he is a teaching assistant in Akita Prefectural University, Japan. His research interests include computer vision, pattern recognition, and machine learning.

Kazuki Saruta received his BE and ME degrees from Akita University, Japan, in 1991 and 1993, and his Ph.D degree from Tohoku University, Japan, in 1996. Then, he was a lecturer in the Faculty of Humanities of Yamagata University. In 1999, he was invited as an associate professor in the Department of Electronics and Information Systems of Akita Prefectural University, Japan. He has been engaged in research on pattern recognition and image processing.

Yuki Terata received his BE degree from Akita Prefecture University, Japan, in 1998, and the ME and Ph.D degrees from the same university in 2000 and 2008. He is a research associate in Akita Prefecture University, Japan. He is interested in VR/AR for cognitive psychology of elderly people. 